\title{
Quality of Sleep at the Ward after Cardiothoracic Surgery
}

\author{
Jozefine Pröpper1,2, Richard van Valen1*, Ron T. van Domburg2, Maaike Brunott1, \\ Ad J. J. C. Bogers 1 \\ ${ }^{1}$ Department of Cardio-Thoracic Surgery, Thoraxcenter, Erasmus Medical Center, Rotterdam, The Netherlands \\ ${ }^{2}$ Department of Cardiology, Thoraxcenter, Erasmus Medical Center, Rotterdam, The Netherlands \\ Email: ${ }^{*}$ r.vanvalen@erasmusmc.nl
}

Received 30 April 2015; accepted 9 June 2015; published 12 June 2015

Copyright (C) 2015 by authors and Scientific Research Publishing Inc.

This work is licensed under the Creative Commons Attribution International License (CC BY).

http://creativecommons.org/licenses/by/4.0/

(c) (i) Open Access

\section{Abstract}

Objective: Sleeping problems are among the issues most mentioned by patients after cardiothoracic surgery. These problems can have a negative effect on duration of the hospital stay and recovery. In the ward of our cardiothoracic surgery department, a study was initiated to assess the quality of sleep after cardiothoracic surgery. The primary objective was to investigate the effect of cardiothoracic surgery on the quality of sleep. The secondary objective was to investigate the quality of sleep. Correlations with perioperative factors and related issues such as the type of surgery and medication were sought. Methods: A consecutive prospective cohort study was initiated ( $N=72$ ). The study used validated questionnaires to assess sleep: the Pittsburgh Sleep Quality Index (PSQI), the Epworth Sleepiness Scale (ESS), the Verran Snyder-Halpern Sleep Scale (VSH) and the Factors Influencing Sleep Quality (FISQ). Results: The PSQI showed that the quality of sleep one month after surgery was inferior to the quality of sleep before surgery (p-value: 0.03 ). The efficiency of sleep (time spent in bed) was higher after surgery then before surgery (p-value: 0.01 ). The VSH showed increased impaired sleep on the third night after surgery. The most disruptive factors were not being comfortable in a hospital bed, pain and the noise of medical devices. Conclusions: The quality of sleep after cardiothoracic surgery is worse when compared with the preoperative situation. The chief influencing factors are discomfort in bed, pain and disturbance from medical devices. The use of pain medication does not improve the quality of sleep.

\section{Keywords}

Cardiac Surgery, Thoracic Surgery, Sleep, Sleep Deprivation, Factors Influencing Sleep Quality (FISQ)

\footnotetext{
"Corresponding author.
}

How to cite this paper: Pröpper, J., van Valen, R., van Domburg, R.T., Brunott, M. and Bogers, A.J.J.C. (2015) Quality of Sleep at the Ward after Cardiothoracic Surgery. Open Journal of Nursing, 5, 529-537. 


\section{Introduction}

Although cardiothoracic surgery has led to improvements in life expectancy and quality of life for patients suffering from an array of cardiovascular problems and thoracic diseases, the quality of life after surgery is still negatively influenced by a number of factors. An important aspect of the quality of life is the quality of sleep, defined as a period of inactivity and the absence of consciousness [1]-[3]. Lack of sleep influences the quality of life and well-being of patients [1]. Sleep deprivation, the lower overall sleep time and disturbance of sleep are common problems during either acute or chronic illness [2]. Surgical patients experience more sleeping problems than medical (nonsurgical) patients, with the exception of psychiatric patients [3] [4].

In the Netherlands each year about 19,000 patients undergo cardiac surgery. This kind of surgery is centralized in 16 hospitals. The Department of Cardiothoracic Surgery of the Erasmus University Medical Centre performs 1100 cardiac procedures and 350 thoracic procedures per year.

The department's protocol at the time of this study consisted of sleep medication on request by patients. Daily complaints by patients and a lack of recent literature into this relevant clinical problem constituted the basis of this study.

Earlier studies in patients undergoing cardiac surgery show that sleeping problems after surgery wre a prevalent complaint [5]-[8]. The low quality of sleep and sleep deprivation lead to fatigue, sleepiness and a slower recovery, longer hospital stays and poor quality of life [6] [9]. Furthermore, it has been shown that poor quality of sleep increases the risk for the development of postoperative delirium in thoracic patients. It has also been demonstrated that the quality of sleep is related to a longer stay in hospital after surgical procedures [9]-[11].

The aim of this study was to investigate the effect of cardiac or thoracic surgery on the quality of sleep using four validated questionnaires (PSQI, ESS, VSH and FISQ). The secondary aims were to determine what factors influenced sleep and the effects of hypnotics and pain medication on the quality of sleep. Furthermore, in order to assess objectively the quality of sleep of our patients, a comparison was done with the quality of sleep in the general population.

Cardiac surgery is here defined as any surgical procedure involving the heart and the thoracic aorta, which is performed to correct acquired or congenital defects, replace or repair disease valves or bypass blocked vessels.

Thoracic surgery is here defined as any surgical procedure involving organs with exception of the heart and thoracic aorta located in the thorax or chest.

\section{Methods}

\subsection{Study Setting and Design}

Between January 1, 2013 and May 5, 2013 all consecutive patients meeting the inclusion criteria (>18 years old and capable of reading and answering questionnaires in Dutch) were included in this prospective study. All patients signed for informed consent. The data was collected during the hospital stay of patients in the ward of the Department of Cardiothoracic Surgery and after discharge. There was a total of five measuring moments. Data was collected by using a flowchart which was applied to all patients (Figure 1). The day before surgery (T0) the first questionnaires were answered (PSQI and ESS). After discharge from intensive care and return to the ward at consecutive measuring moments T1 (first day after surgery), T2 (second day after surgery) and T3 (third day after surgery) the ESS, VSH and FISQ questionnaires were answered by patients. Three weeks after surgery all patients received the last questionnaires (PSQI and ESS) by mail at their home addresses (T4). If there was no reply within two weeks, the patients were called to remind them to answer the last questionnaires. The general and surgical details were retrieved from the electronic data management system. A total of 200 patients were asked to participate in this study, and of this population 72 (36\%) patients agreed to participate. The complete study protocol was followed by 48 (67\%) patients of this group. During the clinical period, data (T1, T2 and T3) were not completed by 24 (33\%) patients. The loss of seven (10\%) patients was due to complications, such as delirium, ischemic stroke or critical illness, but the major part of the lost data was due to patients not answering the clinical questionnaires, six (8\%) patients listed pain as the reason for not answering the questionnaires, 2 wo (3\%) were too tired and another six (8\%) patients forgot or were unable to return the needed forms. The outpatient data was retrieved for 56 (78\%) patients. Two (3\%) of the missing patients who were still admitted to hospital did not fit the research protocol. The other patients (19\%) did not return the form despite two attempts to 


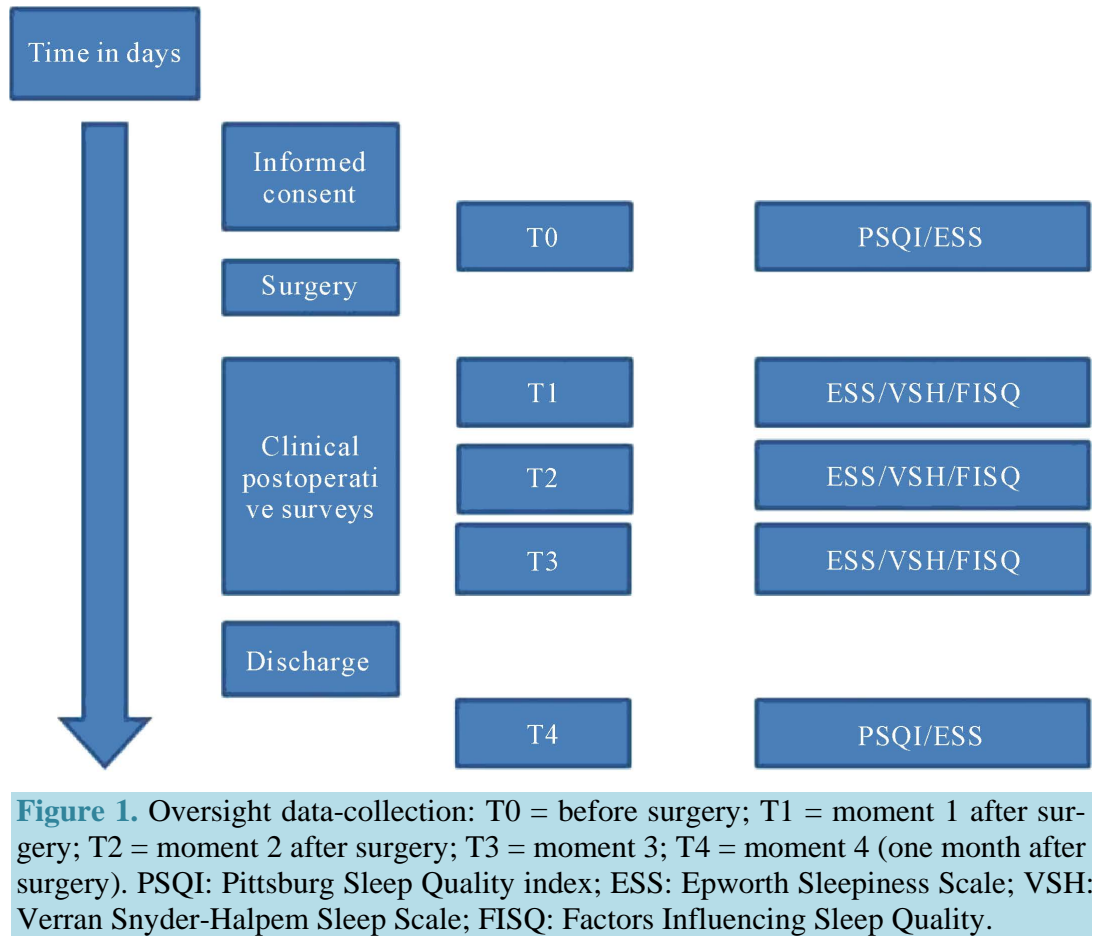

invite them to participate.

\subsection{The Questionnaires}

The following questionnaires were used to assess the quality of sleep in our patient population.

\subsubsection{The Pittsburgh Sleep Quality Index}

The Pittsburgh Sleep Quality Index (PSQI) is a validated questionnaire and investigates different aspects of sleep: the duration of sleep, sleep disturbance, sleep latency, day dysfunction due to sleepiness, sleep efficiency, overall sleep quality and use of sleeping medication [12] [13]. There are also questions for the partner of the patient, but these are not mandatory for obtaining a score. This index is used to interpret quality of sleep during a 1-month period. A higher score indicates a worse quality of sleep.

\subsubsection{The Epworth Sleepiness Scale}

The Epworth Sleepiness Scale (ESS) is also a questionnaire that is validated in Dutch and comprises eight questions to assess sleepiness. This scale was used to assess sleepiness in a clinical environment in an earlier study [14]. A score of more than 10 points indicates sleepiness.

\subsubsection{The Factors Influencing Sleep Quality}

The Factors Influencing Sleep Quality (FISQ) is a questionnaire which is used to determine which variables influence sleep during hospital admission. The scale was validated in 1985 [15] [16] and demonstrated high validity in a previous study about sleep after cardiac surgery [3]. The scale ranges from 0 (no disturbance) to 4 (extreme disturbance). The maximum score possible is 3.54 , and the lowest possible score is 0.71 .

\subsubsection{The Verran Snyder-Halpern Sleep Scale}

The Verran Snyder-Halpern Sleep Scale (VSH) is a scale which has been used in several studies of the quality of sleep of cardiothoracic surgery patients [17]. It comprises of 15 questions and measures the disturbance of sleep, efficacy of sleep and need for additional sleep (sleeping during day-time). The scale was validated in 1987. For the disturbance of sleep the scale ranges from 0 (good) to 70 (bad), for efficacy of sleep from 0 (bad) to 40 (good) and need for additional sleep from 0 (good) to 40 (bad). 


\subsection{Statistical Methods}

The Statistical Package for Social Sciences version 20 (SPSS) was used for all statistical analysis. Variables were compared using an unpaired Student's t-test. A Chi-square test was used for categorical variables. Data are presented as percentages unless indicated otherwise. A p-value $<0.05$ was thought to represent statistical significance. ANOVA for repeated measurements were used for data collected at sequential measuring moments.

\subsection{Ethics}

The local Medical Ethics Committee of the Erasmus Medical Centre approved the study (MEC 2012-576).

\section{Results}

The population predominantly consisted of males (81\%) undergoing cardiac surgery. The most prevalent comorbidities among participating patients consisted of hypertension (35\%), diabetes mellitus type II (15\%) and previous myocardial infarction (13\%) (Table 1). There was hardly any preoperative use of hypnotics (1\%) or anxiolytics (6\%).

\subsection{PSQI}

The quality of sleep four weeks post-surgery was rated lower than the quality of sleep before surgery (p-value: 0.03). This is calculated by comparing the total score on the day before surgery with that on Day 30 after surgery. The sleep efficiency (time spent in bed) at four weeks was higher in comparison to T0 (p-value: 0.01; Figure 2). When comparing the results of this study cohort to the results of the control group in the original validating cohort study [12], patients before and up to one month after surgery scored worse than the control group (the control group total score was 2.67 and the study group total scores were 5.9 and 6.7 at $\mathrm{T} 0$ and T4).

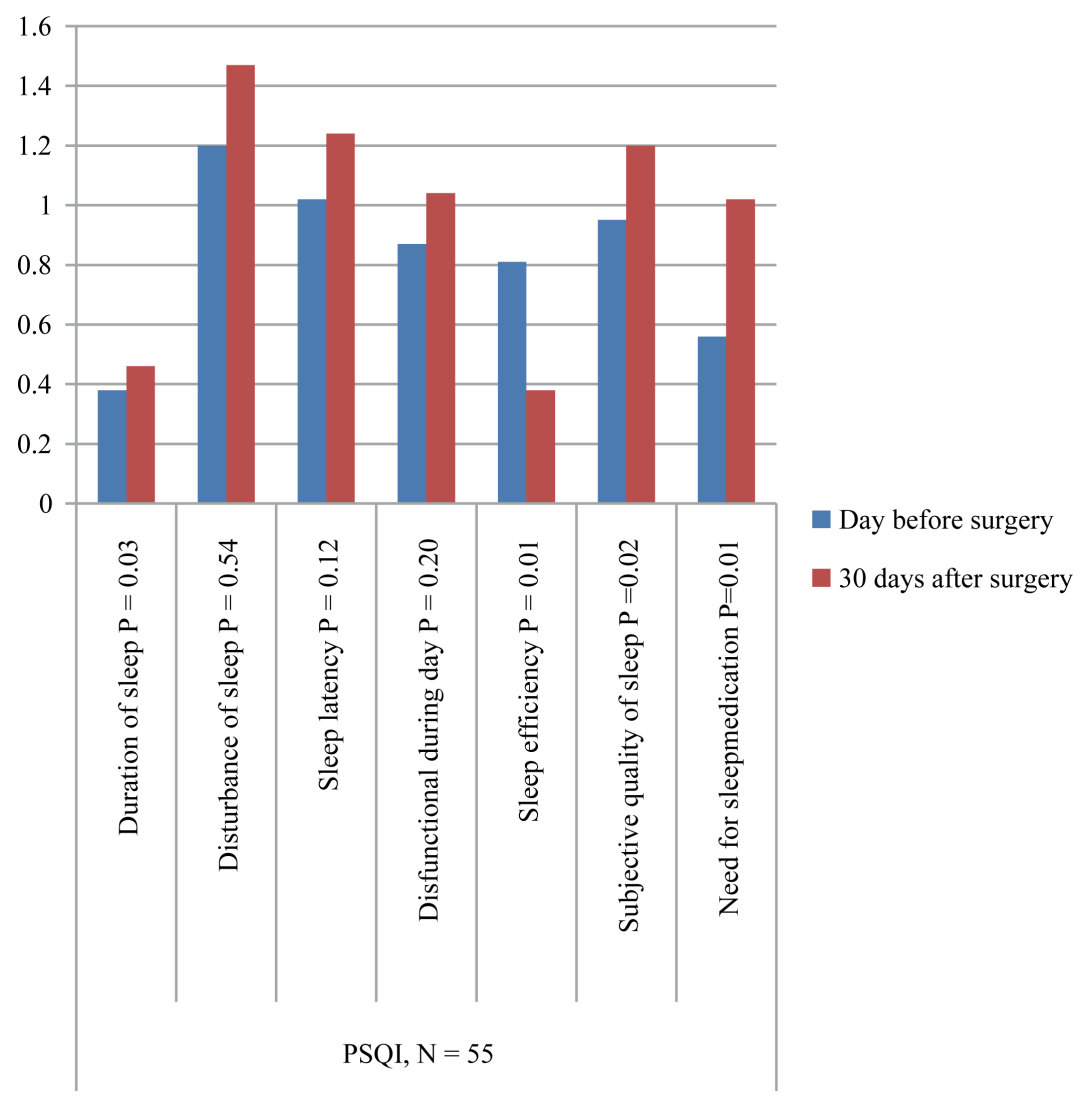

Figure 2. PSQI scores at the day before surgery and 30 days after surgery. Higher scores mean poorer quality of sleep. PSQI: Pittsburg Sleep Quality Index. 
Table 1. Demographic characteristics of patients. SD: Standard deviation, $\mathrm{N}$ : number of patients.

\begin{tabular}{|c|c|}
\hline \multicolumn{2}{|l|}{ Patient Characteristics (\%) } \\
\hline Male & 81 \\
\hline Age, average (SD) & $58(14)$ \\
\hline Body Mass Index, average (SD) & $27(5)$ \\
\hline \multicolumn{2}{|l|}{ Medical History } \\
\hline Atrial fibrillation & 14 \\
\hline Other cardiac aritmia & 1 \\
\hline Chronic obstructive pulmonary disease & 8 \\
\hline Obstructive sleep apneu syndrome & 3 \\
\hline Other sleep disorders & 1 \\
\hline Diabetes mellitus & 15 \\
\hline Mycardial infarction & 13 \\
\hline Alcohol abuse & 1 \\
\hline Heart failure & 4 \\
\hline Hypertension & 35 \\
\hline Psychiatric history & 0 \\
\hline Cerebro-vasculair incident or transcient ischemic accident & 10 \\
\hline Renalin sufficiency & 7 \\
\hline Previous cardiac surgery & 10 \\
\hline Previous thoracic surgery & 1 \\
\hline \multicolumn{2}{|l|}{ Perioperative Factors } \\
\hline Endocarditis & 1 \\
\hline Mediastinitis & 0 \\
\hline \multicolumn{2}{|l|}{ Reason of Admittance } \\
\hline Cardiac surgery & 82 \\
\hline Lung surgery & 14 \\
\hline Other cardio-thoracic surgery & 4 \\
\hline \multicolumn{2}{|c|}{ Previous Used Sleep Medication, Antipsychotics or Anxiolytica } \\
\hline Sleep medication & 1 \\
\hline Antipsychotic medication & 3 \\
\hline Anxiolytica & 6 \\
\hline
\end{tabular}




\subsection{ESS}

This score did not deviate during the five different measurements and was within normal parameters according to the ESS (Table 2).

\subsection{FISQ}

The FISQ (Table 3) showed that the most disturbing factors of sleep were: Not being able or allowed to find a comfortable position in bed, pain and alarm signals from medical equipment.

\subsection{VSH}

The results of the VSH showed that the worst overall quality of sleep occurred during the third night after surgery (T3). Disturbances of sleep were mainly seen on the first night after surgery (T1). This is shown in Table 4. Since pain medication is often used in this patient group, scores were divided into scores with and without pain medication [18].

This study also investigated the effect of pain medication and hypnotics on the quality of sleep. Pain medication was the most frequently administered medication (morphine in combination with acetaminophen). On T1 52\% of all patients received pain medication, on T2 12\% of patients still received pain medication and on the night of T3 10\% received pain medication. There seems to be a trend towards better quality of sleep after pain medication, though this is accompanied by a poorer efficacy of sleep. Analyses of VSH and ESS did not show any significant differences ( $\mathrm{p}$-values $>0.1$ ).

The administration of sleep medication exhibited an increase from $6 \%$ on $\mathrm{T} 1$ to $14 \%$ on $\mathrm{T} 2$ and then to $25 \%$ on T3 (Table 5). Patients receiving sleep medication scored worse on the quality of sleep compared to respondents not using sleep medication. Patients were also scoring lower on efficacy of sleep and number of disturbances. However, there was no difference in sleepiness during the day.

Table 2. ESS: Epworth Sleepiness Scale, N: number, MIS: missing data, SD: standard deviation. A score of $>10$ indicates sleepiness.

\begin{tabular}{|c|c|c|c|c|c|}
\hline ESS & $\begin{array}{c}\text { T0 } \\
\mathrm{N}=73 \\
\mathrm{MIS}=4\end{array}$ & $\begin{array}{c}\mathrm{T} 1 \\
\mathrm{~N}=48 \\
\mathrm{MIS}=0\end{array}$ & $\begin{array}{c}\mathrm{T} 2 \\
\mathrm{~N}=48 \\
\mathrm{MIS}=0\end{array}$ & $\begin{array}{c}\text { T3 } \\
\mathrm{N}=47 \\
\mathrm{MIS}=0\end{array}$ & $\begin{array}{c}\mathrm{T} 4 \\
\mathrm{~N}=56 \\
\mathrm{MIS}=1\end{array}$ \\
\hline Notsleepy, N (\%) & $65(90)$ & $44(92)$ & 46 (96) & $43(92)$ & 53 (95) \\
\hline Sleepy, N (\%) & $3(4)$ & $4(8)$ & $2(4)$ & $4(8)$ & $2(4)$ \\
\hline Average (SD) & $3.7 \pm 3.3$ & $4.7 \pm 3.4$ & $4.2 \pm 2.7$ & $4.5 \pm 3.5$ & $3.6 \pm 2.6$ \\
\hline
\end{tabular}

Table 3. FISQ score of $>1.44$ is moderate to excess of disturbance in sleep. Maximum score possible is 3.54 , lowest possible score is 0.71 .

\begin{tabular}{|c|c|c|c|}
\hline & $\mathrm{T} 1$ & $\mathrm{~T} 2$ & T3 \\
\hline Not being comfortable in bed or unable to move & 2.85 & 2.60 & 2.32 \\
\hline Pain & 2.54 & 2.69 & 2.11 \\
\hline Not being able to perform normal bed routine & 2.27 & 1.88 & 1.66 \\
\hline Noise from medical devices & 2.19 & 1.90 & 2.02 \\
\hline Noises from other patients, like coughing, snoring & 2.10 & 1.81 & 1.96 \\
\hline Undergoing medical or nurse procedures & 1.90 & 1.85 & 1.62 \\
\hline Not being in your own bed & 1.85 & 1.79 & 1.77 \\
\hline Bed making noises & 1.79 & 1.65 & 1.72 \\
\hline Noise made by healthcare providers & 1.71 & 1.75 & 1.57 \\
\hline
\end{tabular}


Table 4. Pain medication first night after surgery. VSH (Verran Snyder-Halpem Sleep Scale): Total score: lower = worse. Disturbance of sleep: Higher = worse. Sleep efficiency: lower = worse. Need for extra sleep: Higher = worse. ESS (Epworth Sleepiness Scale) Higher scores indicatie more sleepy.

\begin{tabular}{ccccc}
\hline & T1 (first night) & With pain medication & Without pain medication & p-value \\
\hline \multirow{2}{*}{ VSH } & Total quality of sleep, average, SD & $73.6 \pm 18.9$ & $70.3 \pm 21.3$ & 0.57 \\
& Disturbance of sleep, average, SD & $35.4 \pm 15.2$ & $38.0 \pm 16.1$ & 0.57 \\
& Efficacy of sleep, average, SD & $16.1 \pm 7.6$ & $18.1 \pm 8.0$ & 0.39 \\
& Need for extra sleep, average, SD & $17.0 \pm 7.1$ & $19.8 \pm 7.1$ & 0.19 \\
ESS & Average, SD & $4.6 \pm 2.7$ & $4.7 \pm 4.3$ & \\
\hline
\end{tabular}

Table 5. Effect of sleep medication on VSH and ESS on third night after surgery. VSH (Verran Snyder-Halpem Sleep Scale): Total score: lower = worse. Disturbance of sleep: Higher = worse. Sleep efficiency: lower $=$ worse. Need for extra sleep: Higher $=$ worse. ESS (Epworth Sleepiness Scale) Higher scores indicatie more sleepy.

\begin{tabular}{ccccc}
\hline & T3 (third night) & With sleep medication & Without sleep medication & p-value \\
\hline \multirow{2}{*}{ VSH } & Total quality of sleep, average, SD & $74.3 \pm 20.8$ & $\mathrm{~N}=36$ & 0.12 \\
& Disturbance of sleep, average, SD & $37.2 \pm 16.3$ & $79.3 \pm 18.5$ & 0.15 \\
& Efficacy of sleep, average, SD & $16.2 \pm 11.1$ & $34.1 \pm 13.6$ & 0.23 \\
& Need for extra sleep, average, SD & $14.7 \pm 4.8$ & $14.9 \pm 5.7$ & 0.81 \\
\hline
\end{tabular}

In this study not only cardiac surgery patients but also thoracic surgery patients $(\mathrm{N}=7)$ were included. The overall trend shows better VSH scores for the thoracic surgery patients. However, the small number of thoracic surgery patients included does not allow generalizing about this trend.

\section{Discussion}

This study shows that quality of sleep after cardiothoracic surgery is worse up to one month after surgery compared to the preoperative situation.

Problems with sleep can be measured using VSH, which measures the disturbance of sleep, efficacy of sleep and need to sleep during the day. That most disturbances are seen on the first night after surgery seems logical. Patients are still attached to wound drainage systems and cardiac surveillance equipment and are often physically examined by nurses and doctors. But the question of why patients experience the worst quality of sleep at night 3 (T3) is more difficult to answer. Monitor rounds are less frequent, and wound drainage systems have been removed. One possible explanation is that not receiving additional pain medication (only 10\% of patients still receive pain medication) accounts for the poorer quality of sleep. However, earlier studies in our centre show a decreased need for pain medication at this time [18].

This effect of poorer sleep can be attributed to limitations after surgery (e.g. not being allowed to sleep on your side, or a different daily schedule).

Further analysis using the ESS (a self-reported measure of sleepiness) showed no deviation from the normal range. This result was unexpected. Patients have undergone major surgery with use of hypnotics and pain medication. It was expected that on day 1 and day 2 patients would experience more sleepiness. Objectifying sleepiness could be included in further research using equipment to either measure actual sleep [14] or by using wrist actigraphy [19]. The last technique has been validated for early detection of delirium by registering the amount of movement by patients and may also provide information on the amount of sleepiness and activity during the night.

Furthermore, the study shows that sleep is disturbed by many factors beyond the control of the patient (the 
FISQ questionnaire). Nurses should be aware of the disturbances that they cause during the nightshift. Furthermore, there should be attention to the problems patients have with positioning and the amount of comfort in bed. This study shows that these are persistent problems causing sleep issues.

Interventions in both nurses' behaviour but also the physical aspect of the ward need to be given more attention, and after implementation the results could be studied using the questionnaires of this study.

This study does not incorporate pain scores into the analysis. The department has a nurse driven protocol which has been shown to result in lower pain scores (according to VAS) [18]. The efficacy of pain medication and low VAS scores on the quality of sleep need further investigation. This study shows a trend towards better sleep with pain medication, in contrast with the trend towards worse quality of sleep despite using hypnotics after surgery. This relationship seems contradictory; with less pain a better quality of sleep should be expected.

The ward comprises a high care and medium care facility. The usual pathway is one night of intensive care after surgery and then referral to the medium care facility. However, 24 patients were admitted to the high care facility after several hours in the ICU and before going to the ward. These patients answered T1 several hours earlier. This could affect the quality of sleep as these patients tended to be in greater pain and require more pain medication.

The poorer quality of sleep seems to continue in the complete follow-up. Comparing the scores to the benchmark set for the normal population continued to demonstrate worse scores. This study did not clarify why this cohort of patients continued to score worse.

A number of patients were unable to follow up for the complete study protocol. From the original 72 included patients only 47 filled in T1, T2 and T3. Using the four questionnaires to assess sleep and the protocol within the timeframe proved to be challenging for both patients and nurses. Therefore, there was an amendment after one week in which a flowchart was given to nurses and patients. The VSH proved to be difficult for most patients. Redundancy or similarity in questions prompted patients to skip such questions.

In future studies the usage of the questionnaires will need to be explained more in depth to patients.

\section{Limitations}

This study has several limitations. The number of patients enrolled was limited, and this made it more difficult to generalize the findings of this study to the overall population of cardiothoracic surgery patients. In follow-up studies more patients should be enrolled in order to further investigate sleeping issues after cardiothoracic surgery.

Furthermore, enrolled subjects had problems with filling out the questionnaires. The redundancy or similarity in questions prompted patients to skip questions. Another reason for not following protocol was the number of questionnaires. This was addressed by a protocol amendment in which the patients and nurses involved received a flowchart with information on the required questionnaires and the timeframe of the questionnaires.

\section{Conclusion}

In conclusion, the quality of sleep after cardiac or thoracic surgery is poor. The influencing factors are discomfort in bed, pain and disturbance from medical devices. The use of pain medication does not improve quality of sleep. Patients after cardiac surgery have a poorer quality of sleep than thoracic surgery patients do. Further research into how long these effects continue and if and when patients return to base-line needs to be formulated.

\section{References}

[1] Jensen, D.P. and Herr, K.A. (1993) Sleeplessness. Nursing Clinics of North America, 28, 385-405.

[2] Parish, J.M. (2009) Sleep-Related Problems in Common Medical Conditions. Chest, 135, 563-572. http://dx.doi.org/10.1378/chest.08-0934

[3] Tranmer, J.E., Minard, J., Fox, L.A. and Rebelo, L. (2003) The Sleep Experience of Medical and Surgical Patients. Clinical Nursing Research, 12, 159-173. http://dx.doi.org/10.1177/1054773803012002004

[4] Doğan, O., Ertekin, S. and Doğan, S. (2005) Sleep Quality in Hospitalized Patients. Journal of Clinical Nursing, 14, 107-113. http://dx.doi.org/10.1111/j.1365-2702.2004.01011.x

[5] Edell-Gustafsson, U.M. and Hetta, J.E. (1999) Anxiety, Depression and Sleep in Male Patients Undergoing Coronary Artery Bypass Surgery. Scandinavian Journal of Caring Sciences, 13, 137-143. 
[6] Edell-Gustafsson, U.M., Hetta, J.E. and Aren, C.B. (1999) Sleep and Quality of Life Assessment in Patients Undergoing Coronary Artery Bypass Grafting. Journal of Advanced Nursing, 29, 1213-1220. http://dx.doi.org/10.1046/j.1365-2648.1999.01006.x

[7] Redeker, N.S. (1993) Symptoms Reported by Older and Middle-Aged Adults after Coronary Bypass Surgery. Clinical Nursing Research, 2, 148-159. http://dx.doi.org/10.1177/105477389300200205

[8] Redeker, N.S., Ruggiero, J. and Hedges, C. (2004) Patterns and Predictors of Sleep Pattern Disturbance after Cardiac Surgery. Research in Nursing \& Health, 27, 217-224. http://dx.doi.org/10.1002/nur.20023

[9] Yildizeli, B., Ozyurtkan, M.O., Batirel, H.F., Kuscu, K., Bekiroglu, N. and Yuksel, M. (2005) Factors Associated with Postoperative Delirium after Thoracic Surgery. The Annals of Thoracic Surgery, 79, 1004-1009. http://dx.doi.org/10.1016/j.athoracsur.2004.06.022

[10] Kjolhede, P., Langstrom, P., Nilsson, P., Wodlin, N.B. and Nilsson, L. (2012) The Impact of Quality of Sleep on Recovery from Fast-Track Abdominal Hysterectomy. Journal of Clinical Sleep Medicine, 8, 395-402. http://dx.doi.org/10.5664/jcsm.2032

[11] Koster, S., Hensens, A.G., Oosterveld, F.G., Wijma, A. and van der Palen, J. (2009) The Delirium Observation Screening Scale Recognizes Delirium Early after Cardiac Surgery. European Journal of Cardiovascular Nursing, 8, 309-314. http://dx.doi.org/10.1016/j.ejcnurse.2009.02.006

[12] Buysse, D.J., Reynolds, C.F., Monk, T.H., Berman, S.R. and Kupfer, D.J. (1989) The Pittsburgh Sleep Quality Index: A New Instrument for Psychiatric Practice and Research. Psychiatry Research, 28, 193-213. http://dx.doi.org/10.1016/0165-1781(89)90047-4

[13] Mariman, A., Vogelaers, D., Hanoulle, I., Delesie, L., Tobback, E. and Pevernagie, D. (2012) Validation of the ThreeFactor Model of the PSQI in a Large Sample of Chronic Fatigue Syndrome (CFS) Patients. Journal of Psychosomatic Research, 72, 111-113. http://dx.doi.org/10.1016/j.jpsychores.2011.11.004

[14] Yilmaz, H. and Iskesen, I. (2007) Follow-Up with Objective and Subjective Tests of the Sleep Characteristics of Patients after Cardiac Surgery. Circulation Journal, 71, 1506-1510. http://dx.doi.org/10.1253/circj.71.1506

[15] Topf, M. (1985) Personal and Environmental Predictors of Patient Disturbance due to Hospital Noise. Journal of Applied Psychology, 70, 22-28. http://dx.doi.org/10.1037/0021-9010.70.1.22

[16] Topf, M. (1985) Noise-Induced Stress in Hospital Patients: Coping and Nonauditory Health Outcomes. Journal of Human Stress, 11, 125-134. http://dx.doi.org/10.1080/0097840X.1985.9936749

[17] Liao, W.C., Huang, C.Y., Huang, T.Y. and Hwang, S.L. (2011) A Systematic Review of Sleep Patterns and Factors That Disturb Sleep after Heart Surgery. Journal of Nursing Research, 19, 275-288. http://dx.doi.org/10.1097/JNR.0b013e318236cf68

[18] van Valen, R., van Vuuren, H., van Domburg, R.T., van der Woerd, D., Hofland, J. and Bogers, A.J. (2012) Pain Management after Cardiac Surgery: Experience with a Nurse-Driven Pain Protocol. European Journal of Cardiovascular Nursing, 11, 62-69. http://dx.doi.org/10.1177/1474515111430879

[19] Osse, R.J., Tulen, J.H., Hengeveld, M.W. and Bogers, A.J. (2009) Screening Methods for Delirium: Early Diagnosis by Means of Objective Quantification of Motor Activity Patterns Using Wrist-Actigraphy. Interactive Cardiovascular and Thoracic Surgery, 8, 344-348. http://dx.doi.org/10.1510/icvts.2008.192278 